\title{
CLASSIFICATION OF INTERFEROMETRIC SAR IMAGES BASED ON PARAMETRIC MODELING IN THE FRACTIONAL FOURIER TRANSFORM DOMAIN
}

\author{
Nazli Deniz Cagatay and Mihai Datcu
}

\author{
German Aerospace Center (DLR) \\ Remote Sensing Technology Institute (IMF) \\ 82234 Oberpfaffenhofen, Germany
}

\begin{abstract}
In this paper, the importance of image transformation for parametric modeling of single-look complex (SLC) and interferometric SAR (InSAR) images is emphasized. For SLC images, the real and imaginary parts of the fractional Fourier transform (FrFT) coefficients have already been modeled with generalized Gaussian distribution (GGD). Here, this work is extended for InSAR images. The Kolmogorov-Smirnov (KS) test statistics show that FrFT simplifies the statistical response for both SLC and InSAR images, and helps to achieve more uniform KS statistics over all classes, which is important in order to model the whole database with a single distribution. Moreover, the classification of InSAR images with a feature vector composed of GGD parameters shows a performance comparable to that of a non-parametric feature vector.
\end{abstract}

Index Terms - Fractional Fourier transform (FrFT), generalized Gaussian distribution, interferometric synthetic aperture radar (InSAR), parametric modeling, scene classification.

\section{INTRODUCTION}

Today, automated and fast exploration of large databases becomes very crucial in many fields. Remote sensing is one of them, where many satellite images are being piled up every day. For scene recognition, classification or indexing purposes, each image in the database is represented by a compact feature descriptor. This feature descriptor can be constructed by means of various parametric and non-parametric methods.

As opposed to the optical satellite images, for the SAR and interferometric SAR (InSAR) images, feature extraction is performed on complex-valued images. Moreover, the acquisition geometry of such images plays an important role in the interpretation of the image content, and hence requires special attention during feature extraction. For instance, due to the sub-aperture decomposition property of complexvalued SAR images [1], the traditional wavelet decomposition faces some limitations when applied to such images [2]. On the other hand, chirplet-based fractional Fourier transform (FrFT) is found to be a more appropriate scaling approach capturing the backscattering phenomenon [2].
In [3] and [4], a non-parametric feature extraction approach based on the FrFT coefficients of single look complex (SLC) SAR and InSAR images is utilized for classification, respectively. On the other hand, parametric modeling of FrFT coefficients of SLC image is studied for classification purposes in [5]. In this paper, this work will be extended to InSAR images.

The statistical model for intensity and phase of InSAR images is examined in [6]. However, in this paper, parametric modeling will be carried out in the transform domain.

\section{STATISTICAL RESPONSE IN FRACTIONAL FOURIER DOMAIN}

A 2-D SLC SAR image is represented as $z=|z| \cdot e^{j \psi}$ with its amplitude $|z|$ and phase $\psi$. Then, the InSAR image is defined as $I=z_{1} \cdot z_{2}^{*}=\left|z_{1}\right| \cdot\left|z_{2}\right| \cdot e^{j \phi}$, where $\phi=\psi_{1}-\psi_{2}$ represents the interferometric phase. In this work, the InSAR image is slightly modified as $I_{\bmod }=\sqrt{\left|z_{1}\right| \cdot\left|z_{2}\right|} \cdot e^{j \phi}$, where the geometric mean of the amplitudes of two SLC images is used instead of their multiplication as the amplitude of the InSAR image [4].

Sometimes, it is easier to handle the image in a suitable transform domain instead of the spatial image domain. Due to the chirp-like characteristics of SLC and InSAR images, a chirplet-based FrFT is used in this work as in [3], [4].

The 1-D FrFT decomposes the signal into chirps as

$$
\begin{aligned}
\operatorname{FrFT}_{\alpha}(\xi) & =A_{\alpha} \cdot \exp \left(j \pi \xi^{2} \cot \alpha\right) \\
\cdot \int \exp \left[j \pi\left(-2 x \xi \csc \alpha+x^{2} \cot \alpha\right)\right] f(x) d x & \\
A_{\alpha} & =\frac{\exp [-j(\pi \operatorname{sgn}(\sin \alpha) / 4-\alpha / 2)]}{|\sin \alpha|^{1 / 2}}
\end{aligned}
$$

where $\alpha=p \pi / 2$ is the transform angle, and $0<|p|<2$ is the transform order of the FrFT. If $\alpha=0$ or a multiple of $2 \pi$, FrFT corresponds to the identity operator, and for $\alpha=\pi / 2$ FrFT takes the standard Fourier transform (FT) [7]. For the 2-D case, i.e., for an image, the 1-D FrFT is first applied on one dimension, and then on the other. 
In order to see how the statistical response of the FrFT coefficients changes with the transform order, the probability density functions (pdfs) of real parts of the FrFT coefficients of SLC, InSAR and modified InSAR are plotted in Fig. 1 for two different sample patches. The first sample patch is from an agricultural field, whereas the second sample patch is taken from an industrial facility with strong scatterers.

As it can be seen from Fig. 1, the pdf for the SLC image itself $(p=0)$ has heavier tails for the second sample patch due to the strong scatterers. Also, the tails of InSAR pdfs are heavier than that of the modified InSAR. That is, the statistical response is simplified by modifying the InSAR amplitude. Moreover, the statistical signature of the images changes with the FrFT order. Here, the idea is to search for a general statistical model fitting the image for all transform orders.

\section{PARAMETRIC MODELING}

In this work, generalized Gaussian distribution (GGD) is tested for SLC and modified InSAR images in both image and transform domains. Once the hypothetical distribution is obtained by estimating the distribution parameters, it can be compared with the empirical distribution by means of a goodness-of-fit (GoF) test. For a distribution with good GoF test statistics, the distribution parameter estimates can be used as a feature descriptor in order to represent the image in a large database.

In [5], the real and imaginary parts of FrFT coefficients of SLC images are assumed to follow the GGD with a generalization to the classical central limit theorem. When the pdfs in Fig. 1 are examined, GGD can be proposed to be an appropriate model for the real and imaginary parts of FrFT coefficients of modified InSAR images, too.

The probability density function of GGD is given as

$$
p_{X}(x ; \alpha, \beta, \mu)=\frac{\beta}{2 \alpha \Gamma(1 / \beta)} \exp \left\{-\left(\frac{|x-\mu|}{\alpha}\right)^{\beta}\right\},
$$

where $\Gamma($.$) is the gamma function, and \alpha>0, \beta>0$ and $\mu$ are the scale, shape and location parameters of the GGD, respectively. The distribution parameters can be estimated by the maximum likelihood (ML) method combined with the method of moments (MoM) for the initial estimates [8].

The difference between the parametric modeling of real and imaginary parts of FrFT coefficients of SLC and that of modified InSAR images with GGD is the location parameter, i.e., the mean of the distribution. For SLC images, zero-mean GGD can be considered as an appropriate model for all FrFT orders [5], whereas the mean is non-zero for modified InSAR images for order $p=0$, i.e., no FrFT case.

The estimation steps for zero-mean GGD are explained in [8], and can be generalized for non-zero GGD as follows:

1. Find an initial guess for $\mu$ by means of the first sample (a) Sample patch 1

(Agricultural field)
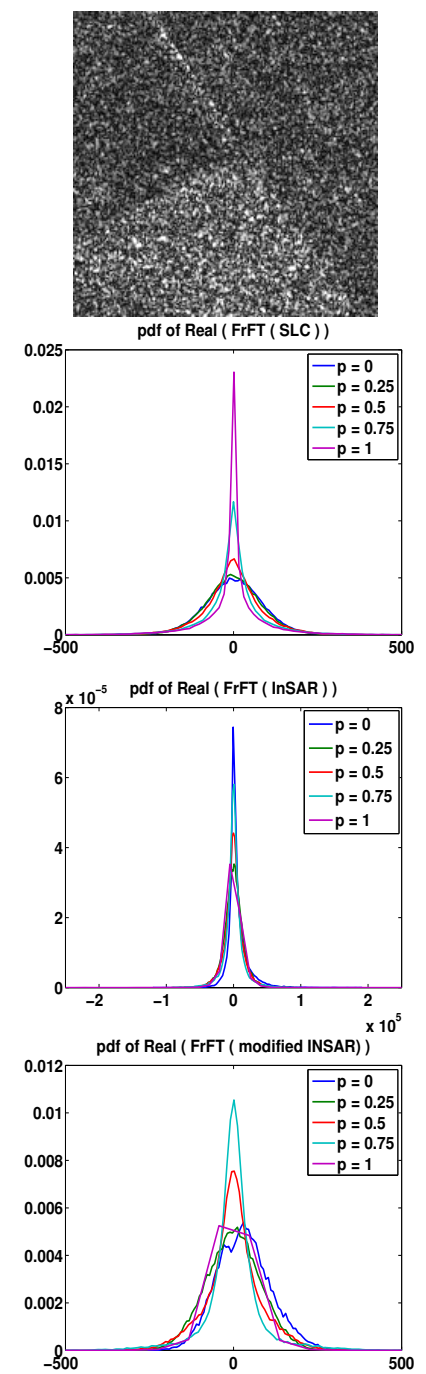

(b) Sample patch 2

(Industrial facility)
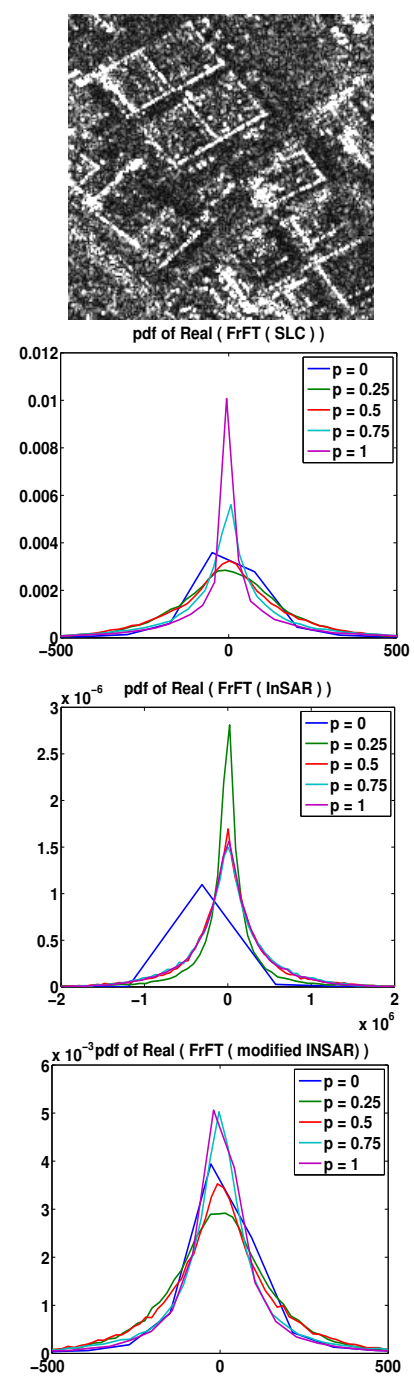

Fig. 1. Pdfs for FrFT domain with different transform orders for 2 different sample patches.

moment:

$$
\hat{\mu}_{0}=\frac{1}{L} \sum_{i=1}^{L} x_{i} .
$$

2. Find an initial guess for $\beta$ by means of the inverse generalized Gaussian ratio function:

$$
\hat{\beta}_{0}=r^{-1}\left(\frac{\hat{m}_{2}}{\hat{m}_{1}^{2}}\right),
$$

where $m_{1}$ is the first central sample moment of the absolute values and $m_{2}$ is the second central sample moment, and calculated as follows:

$$
\begin{aligned}
& \hat{m}_{1}=E\left\{\left|x_{i}-\hat{\mu}_{0}\right|\right\}, \\
& \hat{m}_{2}=E\left\{\left(x_{i}-\hat{\mu}_{0}\right)^{2}\right\} .
\end{aligned}
$$


3. Compute the final estimate $\hat{\beta}$ using the iterative Newton - Raphson method and the initial guess $\hat{\beta}_{0}$ :

$$
\hat{\beta}_{i+1}=\hat{\beta}_{i}-\frac{g\left(\hat{\beta}_{i}\right)}{g^{\prime}\left(\hat{\beta}_{i}\right)},
$$

where $g(\hat{\beta})$ and its derivative $g^{\prime}(\hat{\beta})$ are given in Eq. 8 and Eq. 9 where $\Psi($.$) and \Psi^{\prime}($.$) are the digamma and$ trigamma functions.

4. Final estimate for $\mu$ is found [9]:

$$
\hat{\mu}=\underset{u}{\operatorname{argmin}} \sum_{i=1}^{L}\left|x_{i}-\mu\right|^{\hat{\beta}} .
$$

5. By using the final estimates $\hat{\mu}$ and $\hat{\beta}, \hat{\alpha}$ is found:

$$
\hat{\alpha}=\left(\frac{\hat{\beta}}{L} \sum_{i=1}^{L}\left|x_{i}-\hat{\mu}\right|\right)^{(1 / \hat{\beta})} .
$$

\section{FEATURE EXTRACTION IN FRFT DOMAIN}

In this work, feature extraction is performed in the FrFT domain for 17 different transform angles equally spaced between 0 and $\pi$. The feature vectors (FVs) used in the image classification can be summarized as follows:

- FV-1: Log-cumulants calculated from real and imaginary of $\{\operatorname{FrFT}\{\mathrm{SLC}\}\}$ (FV length $=102)$,

- FV-2: Log-cumulants calculated from real and imaginary of $\{$ FrFT $\{$ modified InSAR $\}\}$ (FV length $=102$ ),

- FV-3: GGD shape and scale parameters estimated from real and imaginary of $\{$ FrFT $\{$ SLC $\}\}($ FV length $=68)$,

- FV-4: GGD shape, scale and location parameters estimated from real and imaginary of $\{$ FrFT \{modified InSAR $\}\}($ FV length $=102)$,

- FV-5: GGD shape and scale parameters estimated from real and imaginary of $\{$ FrFT $\{$ modified InSAR $\}$ \} (FV length $=68$ ).

\section{INSAR IMAGE DATABASE}

For a patch-oriented classification, an image database of total 400 patches with a size of $200 \times 200$ pixels from 8 different classes (50 patches/class) is generated. The classes in the database are as shown in Fig. 2, where $\mathbf{C 1}$ - Agricultural fields, C2 - Forest, C3 - Industrial area, C4 - Mixed vegetation (forest and agricultural), C5 - Riverside, C6 - Urban-1 (with medium density residential area, small houses with gardens), C7 - Urban-2 (high density buildings), C8 - Water body (lakes and ponds).
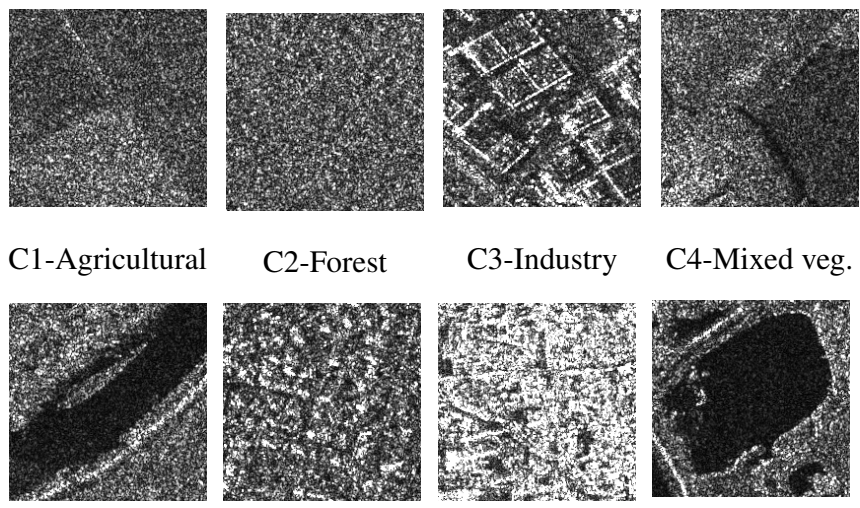

C2-Forest

C3-Industry

C4-Mixed veg.

C5-Riverside
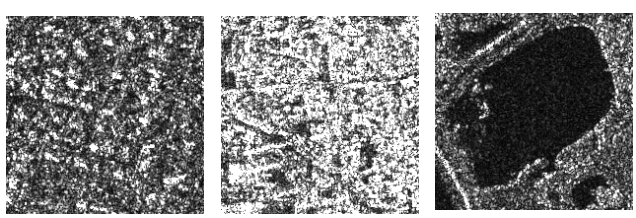

C6-Urban 1

C7-Urban 2

C8-Waterbody

Fig. 2. Classes in the InSAR image database.

\section{RESULTS AND DISCUSSION}

\subsection{Goodness-of-fit test results}

For the given image database, the Kolmogorov-Smirnov (KS) test is used as the GoF test. The KS statistics of all image patches for the GGD are plotted in Fig. 3 for equally spaced 5 different FrFT angles between 0 and $\pi / 2$.

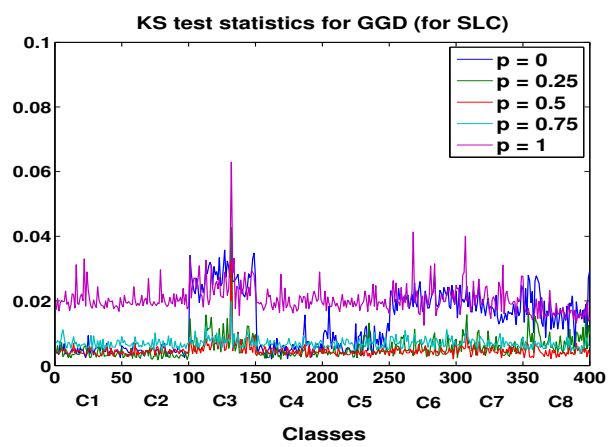

(a)

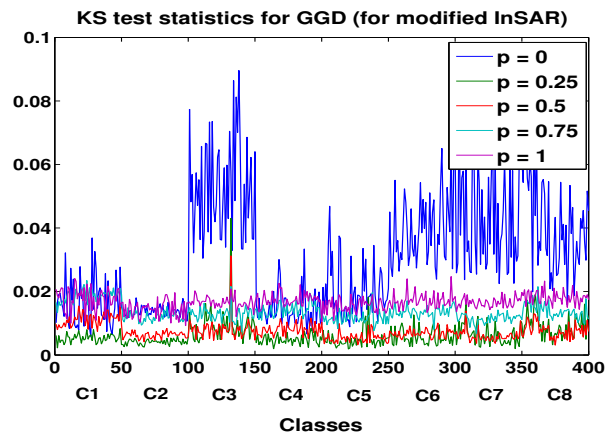

(b)

Fig. 3. KS test statistics for real part of the FrFT coefficients of a) SLC images and b) modified InSAR images for different transform orders $(p=0,0.25,0.5,0.7,1)$. 


$$
\begin{gathered}
g(\hat{\beta})=1+\frac{\Psi(1 / \hat{\beta})}{\hat{\beta}}-\frac{\sum_{i=1}^{L}\left|x_{i}-\hat{\mu}_{0}\right|^{\hat{\beta}} \log \left|x_{i}-\hat{\mu}_{0}\right|}{\sum_{i=1}^{L}\left|x_{i}-\hat{\mu}_{0}\right|^{\hat{\beta}}}+\frac{\log \left(\frac{\beta}{L} \sum_{i=1}^{L}\left|x_{i}-\hat{\mu}_{0}\right|^{\hat{\beta}}\right)}{\hat{\beta}} \\
g^{\prime}(\hat{\beta})=\frac{1}{\hat{\beta}^{2}}-\frac{\Psi(1 / \hat{\beta})}{\hat{\beta}^{2}}-\frac{\Psi^{\prime}(1 / \hat{\beta})}{\hat{\beta}^{3}}-\frac{\sum_{i=1}^{L}\left|x_{i}-\hat{\mu}_{0}\right|^{\hat{\beta}}\left(\log \left|x_{i}-\hat{\mu}_{0}\right|\right)^{2}}{\sum_{i=1}^{L}\left|x_{i}-\hat{\mu}_{0}\right|^{\hat{\beta}}}+\frac{\left(\sum_{i=1}^{L}\left|x_{i}-\hat{\mu}_{0}\right|^{\hat{\beta}} \log \left|x_{i}-\hat{\mu}_{0}\right|\right)^{2}}{\left(\sum_{i=1}^{L}\left|x_{i}-\hat{\mu}_{0}\right|^{\hat{\beta}}\right)^{2}} \\
+\frac{\sum_{i=1}^{L}\left|x_{i}-\hat{\mu}_{0}\right|^{\hat{\beta}} \log \left|x_{i}-\hat{\mu}_{0}\right|}{\hat{\beta} \sum_{i=1}^{L}\left|x_{i}-\hat{\mu}_{0}\right|^{\hat{\beta}}}-\frac{\log \left(\frac{\hat{\beta}}{L} \sum_{i=1}^{L}\left|x_{i}-\hat{\mu}_{0}\right|^{\hat{\beta}}\right)}{\hat{\beta}^{2}}
\end{gathered}
$$

As it can be seen from Fig. 3(a), for SLC images with $p=0$ (no FrFT case), the KS statistics increase for C3, C6 and C7. Besides, for $p=1$, where the FrFT corresponds to the standard FT, the KS statistics increase for all classes. Yet, GGD can be considered as a suitable model for real and imaginary parts of the FrFT coefficients of SLC images with KS statistics around 0.02 and even less for different FrFT orders. On the other hand, from Fig. 3(b), GGD cannot be accepted as an appropriate model for modified InSAR images in image domain, i.e., for $p=0$. However, taking the FrFT, the GGD becomes a more suitable model for modified InSAR with lower KS statistics (values less than 0.02).

In addition, it can be deduced from Fig. 3 that the FrFT helps to simplify the statistical response for both SLC and modified InSAR images, and achieve more uniform KS statistics over all classes in the database. This is important in order to model the whole database with a single distribution.

\subsection{Classification results}

A supervised k-nearest neighbor $(\mathrm{KNN})$ classification with Euclidean distance and $k=1$ is performed on the given database with a randomly chosen training set of $4 \%$. The classification results of 100 runs are averaged and the individual class accuracies are presented in terms of the F-measures.

From Fig. 4(a), it can be seen that FV-3 with GGD parameters improves the individual classification accuracies of $\mathrm{C} 1$, $\mathrm{C} 2$ and $\mathrm{C} 4$ by $25 \%, 46 \%$ and $14 \%$, respectively, whereas the accuracy gets $14 \%$ worse for $\mathrm{C} 3$ compared to FV-1 with logcumulants. When the scene contents are considered, it can be deduced that GGD successfully models scene classes with natural structures, but remains incapable of modeling classes with strong scatterers - consistent with the results of [5].

On the other hand, for modified InSAR images, GGD location parameter deteriorates the classification performance significantly as in FV-4 and it should be omitted from the FV. Then, FV-5, which is composed of GGD shape and scale parameters only, reaches a classification performance comparable to that of FV-2 with log-cumulants, as shown in Fig. 4(b).

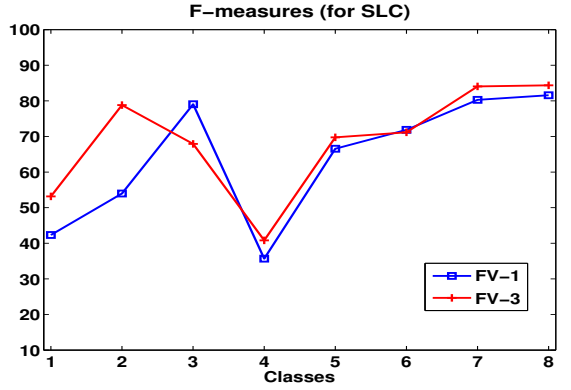

(a)

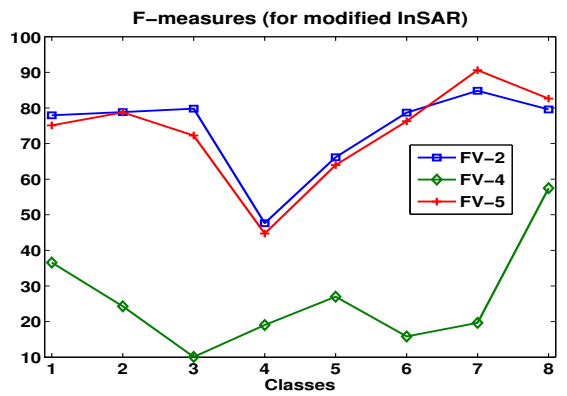

(b)

Fig. 4. KNN classification accuracy results for a) SLC images and b) modified InSAR images.

\section{CONCLUSIONS}

This work extends the parametric modeling of SLC images in FrFT domain to modified InSAR images. For this purpose, the real and imaginary parts of FrFT coefficients of modified InSAR images are modeled with GGD. According to the KS GoF test statistics, although the modified InSAR image itself cannot be appropriately modeled by GGD, FrFT helps to simplify the statistical response and reach a general zero-mean GGD model which is quite suitable for different FrFT orders and different classes. Moreover, KNN classification with a feature vector composed of GGD shape and scale parameters shows a performance comparable to that of a non-parametric feature vector for both SLC and modified InSAR images. 


\section{REFERENCES}

[1] M. Spigai, C. Tison, and J. C. Souyris, "Time-Frequency Analysis in High-Resolution SAR Imagery," IEEE Transactions on Geoscience and Remote Sensing, vol. 49, no. 7, pp. 2699-2711, July 2011.

[2] M. Datcu and J. Singh, "Phase-Scale Analysis of Complex Valued SAR Images," in European Conference on Synthetic Aperture Radar (EUSAR'14), June 2014, pp. 1121-1124.

[3] J. Singh and M. Datcu, "SAR Image Categorization with Log Cumulants of the Fractional Fourier Transform Coefficients," IEEE Transactions on Geoscience and Remote Sensing, vol. 51, no. 12, pp. 5273-5282, December 2013.

[4] N. D. Cagatay and M. Datcu, "Scene Recognition Based on Phase Gradient InSAR Images," in IEEE International Conference on Image Processing (ICIP'14), October 2014, pp. 5162-5166.

[5] J. Singh and M. Datcu, "Parametric Modeling of the Fractional Fourier Transform Coefficients for ComplexValued SAR Image Categorization," in IEEE Inter- national Conference on Image Processing (ICIP'13), September 2013, pp. 2882-2886.

[6] J. S. Lee, K. W. Hoppel, S. A. Mango, and A. R. Miller, "Intensity and Phase Statistics of Multilook Polarimetric and Interferometric SAR Imagery," IEEE Transactions on Geoscience and Remote Sensing, vol. 32, no. 5, pp. 1017-1028, September 1994.

[7] H.M. Ozaktas, O. Arikan, A. Kutay, and G. Bozdagi, "Digital Computation of the Fractional Fourier Transform," IEEE Transactions on Signal Processing, vol. 44, no. 9, pp. 2141-2150, September 1996.

[8] M. N. Do and M. Vetterli, "Wavelet-Based Texture Retrieval Using Generalized Gaussian Density and Kullback-Leibler Distance," IEEE Transactions on Image Processing, vol. 11, no. 2, pp. 146-158, February 2002.

[9] M. K. Varanasi and B. Aazhang, "Parametric Generalized Gaussian Density Estimation," Journal on Acoustical Society of America, vol. 86, no. 4, pp. 1404-1415, October 1989. 